\title{
Perspectivas investigativas en torno a las parejas sin hijos y su relación con el concepto de "familia": Un estado del arte
}

\section{Investigative perspectives on couples without children and their relationship} with the concept of "family": A state of the art

\author{
Germán Arley Baena Vallejoํㅜ, Clara Stella García Quintero ${ }^{2}$, \\ María Cristina Duque Restrepo ${ }^{3}$ y Daniel Stivan Velásquez Muñoz ${ }^{4}$ \\ ${ }^{1}$ Psicólogo. Magister en Paz, Desarrollo y Ciudadanía. Docente e investigador en la Corporación \\ Universitaria Minuto de Dios (UNIMINUTO), Bogotá. E-mail: gbaenava@uniminuto.edu.co \\ ${ }^{2}$ Psicóloga. Magister en Terapia Familiar y de Pareja. Docente e investigadora en la Corporación \\ Universitaria Minuto de Dios (UNIMINUTO), Bogotá. E-mail: cgarciaqui1@uniminuto.edu.co \\ ${ }^{3}$ Trabajadora Social. Especialista en Consultoría Familiar. Magister en Educación. Docente e \\ investigadora en la Corporación Universitaria Minuto de Dios (UNIMINUTO), Bogotá. E-mail: \\ mduquerestr@uniminuto.edu.co
}

${ }^{4}$ Estudiante de Psicología en la Corporación Universitaria Minuto de Dios (UNIMINUTO), Bogotá. E-mail: dvelasque45@uniminuto.edu.co

Trabajo realizado en el marco del proyecto de investigación "Significados de familia en parejas heterosexuales sin hijos", que se lleva a cabo desde agosto de 2018 en la Facultad de Ciencias

Humanas y Sociales de la Corporación Universitaria Minuto de Dios (UNIMINUTO).

Facultad de Ciencias Humanas y Sociales de la Corporación Universitaria Minuto de Dios Bogotá, Colombia

\section{Resumen}

El objetivo del trabajo que se informa fue mostrar cuáles son las transformaciones que las investigaciones manifiestan sobre la familia e identificar si las parejas sin hijos son consideradas como una tipología emergente de configuración familiar. A partir de una investigación documental al modo de estado del arte, se recolectaron 35 artículos entre 1992 y 2017, que se analizaron desde una perspectiva hermenéutica a partir de los núcleos temáticos "definición y transformación del concepto de "familia" y "perspectivas investigativas en torno a la relación entre parejas sin hijos y familia". La mayoría concuerda con que la consanguinidad y la procreación ya no son criterios indispensables para conformar una familia, y así, las parejas sin hijos se constituyen como una nueva tipología familiar que se caracteriza por modos más equitativos, consensuales y democráticos de tomar decisiones; todo esto, bajo el contexto social de una profunda transformación de los roles de género desde el siglo XX.

Palabras clave: parejas sin hijos; familia; transformación; roles de género; procreación. 


\section{Abstract}

The objective of the work reported was to show the transformations the research shows about the family and to identify if couples without children are considered as an emerging typology of family configuration. The importance of the relationship between the categories of family and a couple without children is that this is a time when a significant reduction in the birth rate can be seen worldwide. Furthermore, households made up of couples who state that they do not want to have children also represent a striking increase. On the other hand, this phenomenon has led to the emergence of research categories such as DINK, Childfree and NoMo families, which converge in the increase of couples without children and of women who do not wish to have children.

From a documentary research under the modality of the state of the art, 35 articles were collected for this study between 1992 and 2017, which were analyzed from a hermeneutic perspective from the thematic nuclei "definition and transformation of the concept of "family" and "Research perspectives on the relationship between childless couples and family". The search required the following categories in Spanish and English: "significado de familia/meaning of family", "definición de familia/definition of family", "concepto de familia/family concept", "parejas sin hijos/couple without children/ childfree/NoMo", "nuevas tipologías familiares/new family typologies", "familia DINK/ DINK family" y "familia moderna/modern family". The databases taken into account for the study were Scopus, SciELO, Dialnet Plus, Psicodoc, EBSCO, DOAJ, Redalyc and Google Academic.

Most research agrees that consanguinity and procreation are no longer essential criteria for forming a family, and thus, couples without children are constituted as a new family typology characterized by more equitable, consensual and democratic ways of making decisions. The main reason for this change is the new place that women have conquered in society after decades of struggle for their rights, favoring a questioning of the patriarchal system and the hegemonic model of the family called the nuclear family. The discussions about gender imaginaries that began with greater force in the 20th century, give a place to women in working life that, over time, has favored a questioning about the relationship between motherhood and being a woman.

Research presents different criteria to define the family, which shows that this is a time when there is not one model but several family models. For its part, this diversity has been expressed not only in the dimension of social representations but also in the legal sphere; This is expressed this way, since several investigations in the field of law show transformations in Latin American constitutions by ways of understanding the family in a more plural way and based on fundamental rights.

Finally, the collection of research shows that the study on the category of couples without children is incipient, that is, it corresponds to a phenomenon that, despite enjoying statistical evidence, can be considered emergent in culture insofar as its relevance corresponds to the last decades. Most studies that deal directly or indirectly with the category of childless couple, consider this form of bond as a form of family and explain how at this time this definition is mainly determined by affective criteria, values built between the members, and relational dynamics; leaving in the background the criteria of consanguinity or kinship from the nuclear model.

Keywords: couples without children; family; transformation; gender roles; procreation.

\section{Introducción}

En la época actual se experimenta una llamativa transformación en torno a la institución familiar, distinto a como se la había 
pensado durante varios siglos. Se constata, cada vez más, la idea de que dicho sistema no reside en una estructura o forma de organización única y que le sea natural. La aparición de nuevas formas de organización o tipologías familiares (Grau y Fernández, 2015; Gutiérrez, Díaz y Román, 2016; Martínez y Fernández, 2017; Vásquez, 2005) desde el siglo pasado, no solo ha instalado una duda sobre la unicidad de la estructura familiar, sino que ha dado lugar a un conjunto de debates de interés público que, en último término, han derivado en la necesidad de repensar la definición misma de lo que una sociedad deberá entender por familia.

Estos debates poseen un carácter polémico precisamente porque abren la posibilidad de que la sociedad legitime un conjunto de prácticas que contradicen la concepción tradicional sobre la familia. Por ejemplo, ya en varios países se ha debatido, y en otros apenas ha comenzado, la discusión en torno al matrimonio y la adopción por parte parejas del mismo sexo. En países de Latinoamérica, como Argentina, Brasil, Uruguay y Colombia, esto ha derivado en el establecimiento del derecho de matrimonio entre parejas del mismo sexo que en el mundo ya completa el número de 26 países. Con respecto a la adopción el número asciende a 28 entre los que se encuentran, Argentina, Brasil, Colombia, Uruguay y México.

Estas modificaciones en los modos como las distintas culturas conciben la institución familiar ponen en cuestión la visión tradicional y hegemónica de la familia. Esta visión consiste en la familia nuclear conformada por el padre, la madre y los hijos que han sido resultado del matrimonio de la pareja. Dicha concepción, además, ha estado acompañada por un conjunto de significados sobre las funciones o roles que deben cumplir sus miembros; así, es sabido que al padre se le han asignado funciones relativas al sostenimiento económico y protección de la familia (Martín y Echavarría, 2017), mientras que a la madre se le han asignado funciones de crianza y cuidado del hogar (Gallego, 2012). De los hijos se ha esperado, comúnmente, la incorporación de los valores transmitidos por los padres como base para la consolidación de un sujeto que aporte a la sociedad y que lleve a cabo sus proyectos.

En la concepción tradicional de la familia aparece el matrimonio como uno de los elementos fundamentales que legitima, ante una sociedad, la existencia de dicho vínculo; sin embargo, aparece con fuerza notable el requisito de que la pareja unida en matrimonio pueda procrear para consolidar o completar la estructura familiar (Alberdi, 2016; Fernández, 2017; Lopera, 2016). La función reproductiva de la familia es uno de los aspectos que más se han puesto en cuestión en las últimas décadas (Grau y Fernández, 2015), pues a partir de los eventos históricos en torno a la lucha de las mujeres por situarse en un lugar distinto en la sociedad, y la lucha de los homosexuales por obtener igualdad de derechos respecto a los heterosexuales, la reproducción parece no situarse más como un requisito para ser mujer o para conformar una familia.

Estas transformaciones se conjugan con la emergencia y presencia, cada vez más constante, de parejas que deciden no tener hijos, lo cual da cuenta del establecimiento de una forma de vínculo contemporáneo que se resiste a coincidir con la visión tradicional sobre los vínculos amorosos. El privilegio de los proyectos individuales y de la pareja relativos a la formación académica, las conquistas laborales, los planes turísticos, entre otros, se han soportado en la construcción de nuevas formas de significar los vínculos que han puesto en entredicho la necesidad de tener hijos. En otras palabras, el mandato social que exigía la procreación como consolidación de la familia ha pasado a situarse en un segundo plano para un conjunto de parejas, lo cual ha dado lugar a la construcción de nuevos significados o narrativas sobre lo que es ser familia y sobre la relevancia de la paternidad (Gallardo, Gómez, Muñoz y Suárez, 2006; Nieri, 2017).

No obstante, dada la significación tradi- 
cional de la familia como célula de la sociedad y como institución que cumple la función de socializar a sus descendientes, no es clara la significación o representación que se posee sobre las parejas que deciden no tener hijos en relación al concepto de "familia". Si bien puede apreciarse la emergencia de categorías como la de "Familias DINK" o familias de doble ingreso sin hijos, o la expresión en el ámbito legal denominada "unión de hecho" (Di Brienza, 2007), que no tiene como requisito la presencia de hijos, no es claro el estado actual sobre la investigación ni el abordaje teórico y conceptual que están realizando los autores sobre una forma de vinculación que parece tomar fuerza en la sociedad contemporánea.

En otras palabras, la evidente proliferación de parejas que deciden no tener hijos y su consecuente efecto sobre la significación de la institución familiar generan una inquietud sobre la forma en la que la academia está abordando este fenómeno. Es entonces objeto del presente artículo, mostrar desde una perspectiva de estado del arte el abordaje investigativo al respecto con el fin de reconocer el contenido actual que se le asigna a la relación entre las categorías "pareja sin hijos" y "familia"; por ejemplo, sobre las explicaciones, significados e imaginarios que se instalan desde ámbitos académicos, legales, culturales $\mathrm{y}$, por supuesto, desde la parejas mismas, respecto a este asunto de indagación.

Ahora bien, esta transformación en el concepto de "familia" puede obedecer a la idea según la cual la cultura es una construcción de carácter simbólico que adquiere sus formas de acuerdo a las interpretaciones que sus integrantes le asignan en un momento particular de la historia. Por ejemplo, Martínez (2015) afirma que:

"La cultura no es una unidad monolítica, está formada por un conjunto de imaginarios o significaciones sociales que van dándole sentido a las acciones humanas. Dice Castoriadis que el mundo que creamos a partir de la cultura no es más que una interpretación”. (p. 5)
Este planteamiento se articula con la lectura que hace Corbi (1996) acerca de las modificaciones que ha tenido la religión a partir del periodo de industrialización. Según Corbi, hay una modificación en la relación que el ser humano tenía con la verdad en el periodo preindustrial y la relación que se instala a partir de la segunda industrialización, "para las sociedades preindustriales estáticas, que debían vivir, durante larguísimos períodos de tiempo, de hacer siempre lo mismo, la verdad era lo que Dios había establecido, creado, revelado. Frente a esa verdad no cabía más que la aceptación, la sumisión" (p.35), mientras tanto, "para los hombres de la segunda revolución industrial, que viven de crear nuevos y nuevos conocimientos, la verdad no puede estar ligada a la fijación, al sometimiento, sino que debe estar conectada con la libertad y el dinamismo de crear nuevas y nuevas verdades" (p. 35).

En otras palabras, puede decirse que la concepción tradicional de la familia que es herencia de ese periodo preindustrial amparado especialmente en el cristianismo (Corbi, 1996) obedecía a una forma de relacionarse con la verdad como algo que venía del exterior, es decir, una verdad dada por el mandato divino a la que se debía obedecer. Sin embargo, con el advenimiento de la ciencia y el lugar de la razón en occidente, la relación con la verdad se dinamiza y el ser humano logra ser consciente de su protagonismo en la producción de las formas bajo las cuales lee la realidad. Aunque las concepciones mitológicas que están en la base de la familia nuclear también son creaciones humanas, con el paso del tiempo el ser humano las sacraliza y las convierte en verdades incuestionables que dificultan su modificación (Corbi, 1996; Martínez, 2015); es solo a partir de la vivencia de una profunda crisis en los imaginarios de una sociedad, que se da lugar a nuevas transformaciones en la forma de significar la realidad, y en este caso la estructura familiar.

Este artículo presenta los resultados de un estudio documental a manera de estado 
del arte, sobre las investigaciones que han explorado o de las cuales se puede abstraer la relación entre las categorías de "pareja sin hijos" o "parejas que deciden no tener hijos" y la categoría de "familia". El interés investigativo ha consistido en conocer cuál es el tratamiento que las producciones investigativas le asignan a este tipo de vínculo, con el fin de determinar si es posible denominar a las parejas que deciden no tener hijos como un tipo emergente de configuración familiar.

\section{Metodología}

\section{Postura epistemológica}

Esta investigación se ampara en el paradigma constructivista en tanto se considera que la realidad no es estática, sino que obedece a la construcción simbólica producto del ejercicio interpretativo llevado a cabo por los seres humanos en una cultura y momento histórico particular (Guba y Lincoln, 2002; Guevara, 2016). Como se explicó en el apartado anterior, el concepto de "familia" evidencia en la actualidad una transformación que ha tenido como efecto una redefinición en su configuración y en los significados que la sostienen. De esa construcción sobre la realidad en torno a la familia, participan fenómenos como los imaginarios atávicos (Corbi, 1996; Martínez, 2015), que se ponen en cuestión en la medida en que se van presentando dinámicas sociales (políticas, económica, ideológicas) que exigen modificaciones y que, finalmente, dan cuenta de la maleabilidad inherente de la realidad social.

\section{Estrategia metodológica}

Esta investigación se llevó a cabo a partir de un estudio documental bajo la modalidad de estado del arte. Se entiende por "estado del arte" un estudio riguroso de fuentes bibliográficas sobre investigaciones que abordan el fenómeno en cuestión, no únicamente para describir el conjunto de desarrollos, sino con el fin de conocer las diversas proposiciones teóricas, las metodologías utilizadas, el grado de avance en el abordaje del fenómeno, el rigor y la evidencia en las investigaciones, los vacíos o las contradicciones y, en último término, el conjunto de encuentros y tensiones que pueden establecerse entre las distintas fuentes seleccionadas que denotan una lógica específica en el abordaje teórico y científico del fenómeno hasta la actualidad (Gómez, Galeano y Jaramillo, 2015; Guevara, 2016; Hoyos, 2000; Uribe, 2011; Vélez y Galeano, 2002).

El tratamiento de las fuentes se realizó mediante un ejercicio hermenéutico con el fin de hallar el sentido implícito y explícito de las investigaciones, y de llevar a cabo una lectura intratextual y contextual sobre el fenómeno (Lopera, Ramírez, Zuluaga y Ortiz, 2010). Con respecto a la necesidad del uso de la hermenéutica en los estados del arte, Hoyos (2000) considera que:

"La hermenéutica, en tanto ciencia de la interpretación, es, pues, el elemento estructurador del proceso de construcción de estados del arte; es por ello que, partiendo de la investigación del fenómeno en estudio, se puede llegar a la comprensión y a la transformación del mismo mediante un recorrido que va de los referentes inmediatos a los resultados de esta investigación y finalmente a la comprensión en una secuencialidad que permite enlazar descripción, explicación y construcción teórica nueva desde esa comprensión". (p.33)

\section{Núcleos temáticos y unidades de análisis}

Los núcleos temáticos pueden considerarse como los subtemas o dimensiones de estudio que se desprenden del objeto central de indagación (Guevara, 2016; Hoyos, 2000) y que a su vez, permiten una búsqueda en función de los objetivos específicos de la investigación. En ese sentido, los núcleos temáticos en la presente investigación fueron: definición $\mathrm{y}$ transformación del concepto de familia $\mathrm{y}$ 
perspectivas investigativas en torno a la relación entre parejas sin hijos y familia. El primer núcleo temático ofrece los planteamientos que dan cuenta de cómo las investigaciones están definiendo a la familia y las razones fundamentales que dan cuenta de su transformación. Por su parte, el segundo núcleo temático muestra los planteamientos teóricos que, desde distintas perspectivas investigativas, dan cuenta de cómo se concibe el fenómeno de las parejas sin hijos y si dicho vínculo está siendo considerado como una tipología emergente de la familia.

Las unidades de análisis según Hoyos (2000) corresponden a cada uno de los materiales consultados que permitieron finalmente realizar el análisis de acuerdo a los núcleos temáticos. En total, se recolectaron 35 artículos entre 1992 y 2017 en las bases de datos Scopus, SciELO, Dialnet Plus, Psicodoc, EBSCO, DOAJ, Redalyc y Google Académico. El perfil inicial de los artículos se concentró en cuatro criterios: las bases de datos antes mencionadas, los descriptores de búsqueda según las categorías abstraídas de los objetivos, artículos escritos en inglés y español, y el criterio cronológico de artículos publicados entre los últimos cinco años. Sin embargo, la investigación sobre parejas sin hijos es todavía incipiente, por lo cual fue necesario ampliar los criterios de búsqueda como el año de publicación y las categorías. Por ejemplo, para abstraer algún contenido en torno a la posible concepción de las parejas sin hijos como una modalidad de familia, se decidió incluir categorías más generales que incluyeran todas las tipologías. En ese sentido, las categorías (en español e inglés) utilizadas para realizar la búsqueda fueron: "significado de familia/meaning of family", "definición de familia/definition of family", "concepto de familia/family concept", "parejas sin hijos/ couple without children/childfree/NoMo", "nuevas tipologías familiares/new family typologies", "familia DINK/DINK family" y "familia moderna/modern family".

A continuación, se presenta la Tabla 1, que detalla el perfil inicial de los artículos según su año de publicación, país, tipo de estudio y base de datos. La relación de los artículos con las categorías, bien por mención explícita o mención implícita, están incluidas en la Tabla 2, en el apartado de Resultados.

Tabla 1

Perfil inicial de los artículos.

\begin{tabular}{ccccc} 
Nombre del artículo & Año & País & $\begin{array}{c}\text { Tipo de } \\
\text { estudio }\end{array}$ & $\begin{array}{c}\text { Base de } \\
\text { datos }\end{array}$ \\
\hline Acevedo, L. (2011). El concepto de familia hoy. & 2011 & Colombia & Teórico & $\begin{array}{c}\text { SciELO/ } \\
\text { Dialnet }\end{array}$ \\
\hline $\begin{array}{c}\text { Álvarez, L. (2008). La familia, desarrollo y cambio } \\
\text { social. Claves para un estudio interdisciplinario. }\end{array}$ & 2008 & México & Teórico & $\begin{array}{c}\text { SciELO/ } \\
\text { Dialnet }\end{array}$ \\
\hline $\begin{array}{c}\text { Baeza, S. (2005). Familia y género: Las } \\
\text { transformaciones en la familia y la trama invisible } \\
\text { del género. }\end{array}$ & 2005 & Argentina & Teórico & EBSCO \\
\hline $\begin{array}{c}\text { Benítez, M. (2017). La familia: Desde lo } \\
\text { tradicional a lo discutible. }\end{array}$ & 2017 & Cuba & Reflexivo & DOAJ \\
\hline $\begin{array}{c}\text { Bernal, I. (2013). Juntos aunque separados. Parejas } \\
\text { LAT en la ciudad de Medellín. }\end{array}$ & 2013 & Colombia & $\begin{array}{c}\text { Artículo de } \\
\text { investigación } \\
\text { cualitativa }\end{array}$ & Dialnet \\
\hline
\end{tabular}




\section{Nombre del artículo}

Año

País

Tipo de

Base de

estudio

datos

\begin{tabular}{|c|c|c|c|c|}
\hline $\begin{array}{l}\text { Di Brienza, M. (2007). Modalidad en las uniones } \\
\text { conyugales en Venezuela, continuidad y cambios. }\end{array}$ & 2007 & Venezuela & Reflexivo & $\begin{array}{l}\text { Google } \\
\text { Académico }\end{array}$ \\
\hline $\begin{array}{l}\text { Builes, M. y Bedoya, M. (2008). La familia } \\
\text { contemporánea: relatos de resiliencia y salud } \\
\text { mental. }\end{array}$ & 2008 & Colombia & Reflexivo & $\begin{array}{l}\text { Psicodoc/ } \\
\text { SciELO }\end{array}$ \\
\hline $\begin{array}{l}\text { Cadenas, H. (2015). La familia como sistema } \\
\text { social: Conyugalidad y parentalidad. }\end{array}$ & 2015 & Chile & Teórico & Redalyc \\
\hline $\begin{array}{l}\text { Cadenas, H. y Urquiza, A. (2016). Naturaleza y } \\
\text { contingencia en la familia moderna. }\end{array}$ & 2016 & Chile & Teórico & EBSCO \\
\hline $\begin{array}{l}\text { Cárdenas, M., Coronado, D., Revelo, M., Trujillo, } \\
\text { S. y Gómez, J. (2015). Dinámica relacional de } \\
\text { familias que deciden no tener descendencia. }\end{array}$ & 2015 & Colombia-España & $\begin{array}{l}\text { Artículo de } \\
\text { investigación } \\
\text { cualitativa }\end{array}$ & Dialnet \\
\hline $\begin{array}{l}\text { Chacón, F. y Tapia, M. (2017). No quiero tener } \\
\text { hijos (as)... continuidad y cambio en las relaciones } \\
\text { de pareja de mujeres profesionales jóvenes. }\end{array}$ & 2017 & Chile & $\begin{array}{l}\text { Artículo de } \\
\text { investigación } \\
\text { cualitativa }\end{array}$ & DOAJ \\
\hline $\begin{array}{l}\text { Crespo, F. (2015). Hacia la familia conyugal, } \\
\text { aproximación a los discursos periodísticos en los } \\
\text { siglos XVIII y XIX. }\end{array}$ & 2015 & España & Teórico & Dialnet \\
\hline $\begin{array}{c}\text { Esborraz, D. F. (2015). El concepto constitucional } \\
\text { de familia en América Latina. Tendencias y } \\
\text { proyecciones. }\end{array}$ & 2015 & $\begin{array}{l}\text { Investigación en } \\
\text { Italia sobre América } \\
\text { Latina }\end{array}$ & Teórico & Scopus \\
\hline $\begin{array}{l}\text { Gallego, A. (2012). Recuperación crítica de los } \\
\text { conceptos de familia, dinámica familiar y sus } \\
\text { características. }\end{array}$ & 2012 & Colombia & Teórico & $\begin{array}{l}\text { Redalyc/ } \\
\text { SciELO }\end{array}$ \\
\hline $\begin{array}{l}\text { Garrido, A., Reyes, A., Torres, L. y Ortega, P. } \\
\text { (2008). Importancia de las expectativas de pareja } \\
\text { en la dinámica familiar. }\end{array}$ & 2008 & México & $\begin{array}{c}\text { Artículo de } \\
\text { investigación } \\
\text { cuantitativa }\end{array}$ & Redalyc \\
\hline $\begin{array}{l}\text { González, M. (2009a). Nuevas familias, nuevos } \\
\text { retos para la investigación y la educación. }\end{array}$ & 2009 & España & Teórico & Scopus \\
\hline $\begin{array}{c}\text { González, M (2009b). Familia, un actor con } \\
\text { historia propia. }\end{array}$ & 2009 & Chile & Reflexivo & Dialnet \\
\hline $\begin{array}{c}\text { González, J. (2011). Familia hoy: elementos para } \\
\text { la discusión }\end{array}$ & 2011 & $\begin{array}{l}\text { Brasil, Colombia, } \\
\text { Uruguay, Paraguay } \\
\text { y Chile }\end{array}$ & Teórico & $\begin{array}{l}\text { Dialnet/ } \\
\text { SciELO }\end{array}$ \\
\hline $\begin{array}{c}\text { Grau, C. y Fernández, M. (2015). Relaciones de } \\
\text { parentesco en las nuevas familias. Disociación } \\
\text { entre maternidad/paternidad biológica, genética y } \\
\text { social. }\end{array}$ & 2015 & España & Reflexivo & Dialnet \\
\hline $\begin{array}{c}\text { Guerrero, J. (2016). La familia: realidades y } \\
\text { cambio social. }\end{array}$ & 2016 & España & Reflexivo & Dialnet \\
\hline $\begin{array}{l}\text { Gutiérrez, R., Díaz, K. y Román, R. (2016). El } \\
\text { concepto de familia en México: una revisión desde } \\
\text { la mirada antropológica y demográfica. }\end{array}$ & 2017 & México & Teórico & $\begin{array}{l}\text { Dialnet/ } \\
\text { SciELO }\end{array}$ \\
\hline $\begin{array}{l}\text { Juárez, M. (2007). La familia: algunos cambios } \\
\text { sociales significativos. }\end{array}$ & 2007 & España & Reflexivo & $\begin{array}{l}\text { Google } \\
\text { Académico }\end{array}$ \\
\hline
\end{tabular}




\begin{tabular}{|c|c|c|c|c|}
\hline Nombre del artículo & Año & País & $\begin{array}{l}\text { Tipo de } \\
\text { estudio }\end{array}$ & $\begin{array}{l}\text { Base de } \\
\text { datos }\end{array}$ \\
\hline $\begin{array}{c}\text { Machado (2014). La transformación del concepto } \\
\text { constitucional de familia. Alcances a una } \\
\text { problemática teórica. }\end{array}$ & 2014 & Colombia & Teórico & SciELO \\
\hline $\begin{array}{c}\text { Martínez, L. y Fernández, S. (2017). Nuevas } \\
\text { familias e intervención social: aproximación } \\
\text { conceptual a las nuevas modalidades familiares } \\
\text { monoparentales. }\end{array}$ & 2017 & España & Teórico & $\begin{array}{c}\text { Google } \\
\text { Académico }\end{array}$ \\
\hline $\begin{array}{c}\text { Moreno, A. (2010). La emergencia de una nueva } \\
\text { concepción de la familia entre las y los jóvenes } \\
\text { españoles. }\end{array}$ & 2010 & España & Teórico & $\begin{array}{l}\text { Psicodoc/ } \\
\text { Dialnet }\end{array}$ \\
\hline $\begin{array}{l}\text { Oliva, E. y Villa, V. (2014). Hacia un concepto } \\
\text { interdisciplinario de la familia. }\end{array}$ & 2014 & México & Teórico & SciELO \\
\hline $\begin{array}{c}\text { Palacio, C. (2009). Los cambios y } \\
\text { transformaciones en la familia. Una paradoja entre } \\
\text { lo sólido y lo líquido. }\end{array}$ & 2009 & Colombia & Reflexivo & $\begin{array}{l}\text { Google } \\
\text { Académico }\end{array}$ \\
\hline $\begin{array}{l}\text { Pérez, F. (1995). Las parejas sin hijos en Portugal } \\
\text { y España. }\end{array}$ & 1995 & Portugal & Teórico & Dialnet \\
\hline $\begin{array}{c}\text { Pineda, C. (2007) ¿Las parejas sin hijos viven } \\
\text { mejor? }\end{array}$ & 2007 & México & Reflexivo & EBSCO \\
\hline $\begin{array}{c}\text { Vásquez, C. (2005). Las nuevas tipologías } \\
\text { familiares y los malestares interrelacionales que se } \\
\text { suscitan en ellas. }\end{array}$ & 2005 & Colombia & Reflexivo & Redalyc \\
\hline $\begin{array}{l}\text { Vega, Y. (2010). El nuevo rostro de la familia: } \\
\text { cuadros de una exposición. }\end{array}$ & 2010 & México & Reflexivo & Dialnet \\
\hline $\begin{array}{c}\text { Walters, K., Cintrón, F. y Serrano, I. (2006). Familia } \\
\text { Reconstituida. El Significado de "Familia" en la } \\
\text { Familia Reconstituida. }\end{array}$ & 2006 & Puerto Rico & $\begin{array}{l}\text { Artículo de } \\
\text { investigación } \\
\text { cuantitativa }\end{array}$ & Redalyc \\
\hline $\begin{array}{l}\text { Wong, D. (2015). Asexuality in China's sexual } \\
\text { revolution: Asexual marriage as coping strategy. }\end{array}$ & 2015 & China & Teórico & Scopus \\
\hline $\begin{array}{l}\text { Xu, A. y Xia, Y. (2014). The changes in Mainland } \\
\text { Chinese Families During the Social Transition: A } \\
\text { Critical Analysis. }\end{array}$ & 2014 & China & $\begin{array}{l}\text { Investigativo } \\
\text { de enfoque } \\
\text { cuantitativo }\end{array}$ & Scopus \\
\hline $\begin{array}{l}\text { Zhongxin, S., Zhan H. y Xizhe, P. (2017). The } \\
\text { impact of changes in china's family patterns on } \\
\text { family pension functions. }\end{array}$ & 2017 & China & $\begin{array}{l}\text { Investigativo } \\
\text { de enfoque } \\
\text { cuantitativo }\end{array}$ & Scopus \\
\hline
\end{tabular}

\section{Instrumento}

Para el análisis de la información se hizo uso de la matriz categorial que consiste en un instrumento elaborado en Excel en el que se especifica el nombre del artículo, los autores, el propósito o la pertinencia del material, las categorías a las que aporta el artículo, las citas más relevantes, un comentario analítico sobre cada artículo, un resumen y la base de datos de la cual fue recuperado.

\section{Procedimiento}

Se siguieron las fases planteadas por Hoyos (2000) para los estados del arte, que se describen a continuación:

\section{Fase preparatoria}

De acuerdo con Hoyos (2000), esta fase pretende orientar mediante sustento teórico "cuál es el objeto de investigación que se 
pretende abordar, cuáles los núcleos temáticos comprendidos en el tema central; cuál es el lenguaje básico común a utilizar, así como los pasos a seguir a través de la investigación" ( $p$. 40).

\section{Frase descriptiva}

Esta fase consiste en la recolección de las fuentes documentales a partir de los núcleos temáticos y categorías establecidas en la primera fase. También implica la organización de la información con el fin de reconocer a los autores, las metodologías, las posturas conceptuales, los referentes teóricos, entre otros.

\section{Fase interpretativa}

Esta fase "permite ampliar el horizonte de estudio por unidad de análisis y proporciona datos nuevos integrativos por núcleos temáticos, en tanto trasciende lo meramente descriptivo que conduce al planteamiento de hipótesis o afirmaciones útiles para la siguiente fase" (Hoyos, 2000, p. 41). En esa vía, durante cada reunión se presentó la lectura y análisis de entre tres y cuatro artículos para ser discutidos por todos los investigadores. Las discusiones se plasmaron mediante actas donde se sintetizan los elementos principales que también fueron utilizados para la redacción de resultados.

\section{Fase de construcción teórica global}

Según Hoyos (2000), esta fase lleva a cabo una “interpretación por núcleo temático, para mirar los resultados del estudio como vacíos, limitaciones, dificultades, tendencias y logros obtenidos con el fin de presentar el estado actual de la investigación de manera global" (p. 41). En esta fase no se contempla únicamente la elaboración del presente artículo, sino también de los comentarios analíticos en la matriz categorial y las actas respecto a las discusiones de los artículos.

\section{Resultados y discusión}

El análisis de los artículos hallados en las bases de datos arrojó los resultados que se presentan a continuación. Se plantean aspectos que coinciden en las distintas investigaciones $\mathrm{y}$, a la vez, los puntos de desencuentro o en tensión que denota el estado actual de la investigación sobre las parejas sin hijos y su relación con el concepto de "familia". Este apartado se presenta a partir de las dos dimensiones de estudio o núcleos temáticos en los que se concentró el estado del arte, a saber, la definición y transformación del concepto de "familia", y las perspectivas investigativas en torno a la relación entre las parejas sin hijos y la familia.

\section{Definición y transformación del concepto de "familia"}

A diferencia de las parejas sin hijos, la familia es una categoría muy estudiada desde diferentes campos disciplinares de las ciencias sociales. Un punto en común que se halla en la mayoría de artículos es que la definición de "familia" no obedece a criterios únicamente biológicos o naturales, sino que en su concepción inciden factores de carácter social que hacen a la familia un sistema interdependiente de otros como el país, la economía, las políticas estatales, entre otros (Builes y Bedoya, 2008). Sin embargo, estos factores no afectan a las familias de forma homogénea, sino que interaccionan generando un tipo particular de organización. Para Oliva y Villa (2014), cada familia es única y diferente, no solo por las relaciones, los roles y el número de personas que la conforman, sino también por las actividades y los trabajos que realizan o la manera en que se organizan y proyectan. En ese sentido, la familia "no puede ser estudiada como una institución inmutable y tradicional, se requiere que de manera continua se reconsidere su forma y definiciones bajo las nuevas dinámicas" (Oliva y Villa, 2014, p. 13).

Las investigaciones muestran caracterís- 
ticas que predominan en su forma de definir a la familia, por ejemplo, algunos consideran que dicha institución se define por la utilidad que le presta a la sociedad, la generación de vínculos de solidaridad y por representar un modelo de sociedad política (Oliva y Villa, 20014). Otros la definen por su capacidad para favorecer el desarrollo humano de sus integrantes $\mathrm{y}$, con ello, el desarrollo de las instituciones y las regiones (Álvarez, 2008). En otros casos, porque favorece la adaptación de sus integrantes y el establecimiento de lazos afectivos (Crespo, 2015). Por su parte, para Cadenas y Urquiza (2016), la familia es definida como "un sistema de comunicación humana, cuya función es incluir personas en su totalidad bajo la forma de parientes en un entramado de expectativas de conducta recíproca" (p. 94). Finalmente, otros mantienen como elementos básicos la presencia de la conyugalidad y la parentalidad (Cadenas et al., 2015).

De estas definiciones se pueden plantear dos aspectos: en primer lugar, las investigaciones están trascendiendo el criterio clásico de consanguinidad presente en las definiciones tradicionales sobre la familia y que conformaba uno de los elementos provenientes de la definición clásica establecida por Claude Lévi-Strauss (Benítez, 2017; Builes y Bedoya, 2008; Cadenas, 2015; Cadenas y Urquiza, 2016; Oliva y Villa, 2014). Como se puede apreciar, aparecen otros criterios que trascienden lo biológico para dar mayor relevancia a la construcción de los vínculos, las funciones de los miembros y lo que el sistema le proporciona a sus integrantes en relación consigo mismos y con la sociedad. En segundo lugar, es evidente que no se puede llegar a un consenso en las investigaciones con respecto a una sola definición sobre la familia; sus diversas características impiden encasillarla bajo una única forma de concebirla, su carácter es plural y se construye en la interacción de múltiples factores, tanto intrínsecos como extrínsecos al sistema (Builes y Bedoya, 2008).
La definición clásica sobre la familia es mucho más común y susceptible de consenso entre los artículos consultados. En ella hay tres aspectos diferenciadores: en primer lugar, la familia era concebida como la célula o núcleo básico de la sociedad (Acevedo, 2011; Benítez, 2017; Esborraz, 2015; González, 2009b). En segundo lugar, la familia ideal, más conocida como "familia nuclear", debería estar conformada por el padre, la madre y los hijos (Baeza, 2005; González, 2011; Guerrero 2016; Oliva y Villa, 2014). En tercer lugar, deben participar los criterios de consanguinidad, conyugalidad y parentalidad, y el propósito reproductivo generacional es indispensable (Baeza, 2005; Cadenas, 2015; González, 2009b).

¿Qué favoreció la transformación de esa visión ideal y clásica de la familia a una concepción pluralista y que ha dado lugar a la transición descrita por González (2009a) a través de la expresión "de la familia modelo a los modelos de familia" (p. 382)? Como lo señalan Gutiérrez, Díaz y Román (2016), en la etimología de la palabra "familia" se hace alusión a una unidad conformada por un jefe varón y los esclavos (esposa e hijos) que se remonta a la antigüedad grecorromana. Esa concepción se mantuvo, aunque con modificaciones, durante la Edad Media bajo la ideología cristiana mediante la cual se acentuó la idea una familia nuclear en la que la mujer seguía ocupando un lugar inferior al del hombre. De acuerdo con Baeza (2005), el advenimiento de la revolución industrial y el surgimiento del capitalismo certificaron "la división social y sexual del trabajo = hombre productor de bienes, mujer reproductora social de la especie; el mundo queda dividido en un mundo social y un mundo doméstico" (p. 36). Sin embargo, el acceso a la educación $\mathrm{y}$ al trabajo por parte de las mujeres parecen ser los eventos trascendentales en lo que podría denominarse la génesis de la transformación de la familia (Chacón y Tapia, 2017). Las mujeres accedieron a estos dos derechos a finales del siglo XIX, lo que posteriormente daría lugar a la primera ola del feminismo en 
el siglo XX y que inició las reclamaciones por la igualdad de derechos respecto al hombre en asuntos como el trabajo, el matrimonio o el sufragio.

La incidencia de estos eventos es notable para la transformación de la familia ya que la problematización del género pone en discusión la división social de los roles hombre/ proveedor, mujer/ama de casa. El surgimiento de los movimientos feministas trajo consigo una modificación en los imaginarios de la familia patriarcal relativos a la mujer como, por ejemplo, su inferioridad ante el hombre, su asexualidad y su función materna (Baeza, 2005), que dio lugar al establecimiento de otros imaginarios como la complementariedad de los roles (Baeza, 2005), la autonomía de la mujer (Chacón y Tapia, 2017) e incluso, al cuestionamiento del modelo tradicional sobre la masculinidad asociado a términos como "poder", "fuerza", “éxito", “omnipotencia” y "autosuficiencia", entre otros (Baeza, 2005).

A estos eventos históricos en torno al lugar de la mujer, se le suman las investigaciones en el campo del derecho (Esborraz, 2015; Machado, 2014; Vega, 2010) que analizan las transformaciones constitucionales que, a la vez, han legitimado las transformaciones de la familia a lo largo de la historia. En estas investigaciones puede apreciarse que, a partir de una oleada de reformas constitucionales, especialmente a partir de la década de 1980, los estados latinoamericanos realizaron un viraje a la concepción y legislación sobre la familia. El aspecto fundamental que permitió esa modificación según Esborraz (2015) es que la Constitución se empezó a considerar como un "mandato directo del poder constituyente al poder constituido, cuya resultante es la 'constitucionalización' e 'internacionalización' de todo el ordenamiento jurídico" (p. 50); en consecuencia, las constituciones obedecieron los mandatos o acuerdos internacionales en torno a los Derechos Humanos, que implicaron "una relectura de las relaciones familiares a la luz de los derechos fundamentales" (2015, p. 50).
Estos acontecimientos en el contexto de la legislación estatal han sido trascendentales, pues como menciona Esborraz (2015):

"En la mayor parte de los ordenamientos de Latinoamérica, aunque con diferentes matices, se ha pasado: i) de un modelo de familia 'totalizante' a otro más 'democrático', donde se trata de conciliar el interés familiar con el interés personal de sus integrantes (sobre todo de aquellos más vulnerables), y ii) de un modelo 'único' de familia al reconocimiento de una 'pluralidad' de modelos familiares, todos ellos dignos de igual tutela (como pueden ser, p. ej., la familia matrimonial, la convivencial, la homoparental, la monoparental, la ensamblada, la ampliada, la indígena, etc.)". (p. 50)

Ahora bien, un punto que puede hallarse en tensión es que a diferencia de aquellos que hablan de una crisis de la familia (Acevedo, 2011) o de una pérdida de los valores, otros (González, 2009a; Guerrero, 2016; Oliva y Villa, 2014; Vega, 2010; Walters, Cinrtrón y Serrano, 2006) son enfáticos en tener en consideración la mutabilidad de la familia, pues es esta característica la que ha permitido que la forma en la que se organiza y se soporta ese sistema varíe de acuerdo a las "necesidades y aspiraciones de los seres humanos en distintos contextos y momentos" (González, 2009a, p. 383). Dicha mutabilidad no responde a lo que algunos han denominado como una "pérdida de valores, pues más bien se trata de un cambio y renovación de ellos (Walters et al., 2006); la cultura y los movimientos que se han presentado en las últimas décadas ha favorecido la discusión sobre:

"Valores de desigualdad o jerarquía de poder, en los que se basaba típicamente la familia patriarcal, pero sigue estando vigente el valor de la solidaridad, que se ve acompañado por nuevos valores familiares, como la igualdad, la libertad, la individualidad, la tolerancia o la felicidad, valores que serán característicos no solo de las nuevas familias sino, de modo más general, reflejo de nuevas formas de orden social". (González, 2009a, p. 383) 
El contexto cultural actual demanda, según González (2009a), una posición diferente por parte de los investigadores, pues sus posturas teóricas y metodológicas se han amparado en un modelo o patrón de familia que hoy no se sostiene. En ese sentido, la autora sugiere hacer una revisión de los métodos, las técnicas y los modelos teóricos con el fin de responder a la emergencia de las nuevas realidades familiares. En su argumentación, González muestra cómo algunos estudios se enfocan en conocer los aspectos negativos de las nuevas organizaciones familiares o algunas técnicas utilizadas desestiman en su formulación de preguntas la existencia de parejas homosexuales, mientras que otras técnicas utilizadas ofrecen formas de puntuación que se amparan en posturas patriarcales (2009a).

Para ir concluyendo este apartado, probablemente uno de los autores consultados que mejor sintetiza las transformaciones de la familia en la actualidad sea Guerrero (2016), quien destaca:

“a) la postnuclearización de la familia, b) la desinstitucionalización del matrimonio, c) la redefinición de los roles de género en el seno de la familia, d) la comprensión de la pareja desde la dimensión afectiva de la vida y, por último, e) la inclusión en el proyecto familiar y de pareja de valores individualistas y de una ética relativista.". (p.159)

En síntesis, podría decirse que la transformación de la familia, que ha devenido en una multiplicidad de formas de definirla, ha estado favorecida por eventos de carácter histórico en los que la mujer ha jugado un papel determinante en tanto ha puesto en cuestión los roles asociados a los géneros, lo cual ha devenido en una profunda transformación de las dinámicas sociales en las que por supuesto inciden aspectos económicos (como la misma dinámica del sistema) o políticos (como las luchas y las conquistas constitucionales) (Moreno, 2010). Lo que es claro, según Juárez (2007), es que estas transformaciones dan lugar, cada vez más, a una concepción pluralista sobre la familia que muestra un posible tránsito de las sociedades autoritarias a la construcción de sociedades democráticas.

\section{Perspectivas investigativas en torno a la relación entre parejas sin hijos y familia}

A continuación se presenta la Tabla 2, en la que se muestran algunas relaciones sobre los artículos recolectados y sobre las cuales se tratará en este apartado.

\section{Tabla 2}

Características de los artículos respecto a la relación pareja sin hijos/familia.

\section{Criterio Cantidad de artículos}

Artículos recolectados sobre la familia que hacen alusión implícita o explícita a la categoría "pareja sin hijos".
18 de 35 recolectados
Baeza (2005); Benítez (2017); Bernal (2013); Cadenas (2015); Cárdenas, Coronado, Revelo, Trujillo y Gómez (2015); Chacón y Tapia (2017); Esborraz (2015); Garrido, Reyes y Torres (2008); González (2011); Guerrero (2016); Juárez (2007); Palacio (2009); Pérez (1995); Pineda (2007); Vega (2010); Wong (2015); Xu y Xia (2014); Zhongxin, Zhan y Xizhe (2017).
Artículos sobre la familia que mencionan explícitamente a la pareja sin hijos como una modalidad de familia pero no lo desarrollan.
Baeza (2005); Cárdenas, Coronado, Revelo, Trujillo y Gómez (2015); Chacón y Tapia (2017); Garrido, Reyes y Torres (2008), González (2011); Guerrero (2016); Juárez (2007); Palacio (2009); Pineda (2007); Pérez, (1995); Vega (2010); Wong (2015); Xu y Xia (2014); Zhongxin, Zhan y Xizhe (2017). 


\section{$\begin{array}{lcc}\text { Criterio } & \begin{array}{c}\text { Cantidad } \\ \text { de artículos }\end{array} & \text { Autores }\end{array}$}

\begin{tabular}{lll}
\hline $\begin{array}{c}\text { Artículos sobre la familia que } \\
\text { mencionan implícitamente a la pareja } \\
\text { sin hijos como una modalidad posible }\end{array}$ & 1 & Benítez (2017).
\end{tabular}
de familia.

\begin{tabular}{|c|c|c|}
\hline $\begin{array}{l}\text { Artículos sobre la familia que no } \\
\text { consideran a la pareja sin hijos como } \\
\text { una modalidad de familia. }\end{array}$ & 3 & $\begin{array}{l}\text { Bernal (2013); Garrido Reyes y Torres (2008); Pérez } \\
\text { (1995). }\end{array}$ \\
\hline
\end{tabular}

Artículos que tienen como tema central la categoría "pareja sin hijos".
Cárdenas, Coronado, Revelo, Trujillo y Gómez (2015); Chacón y Tapia (2017); Garrido, Reyes y Torres (2008); Pineda (2007); Pérez (1995).
Artículos que tienen como tema central la categoría "pareja sin hijos" y consideran este tipo de vínculo como una modalidad de familia.
5

Cárdenas, Coronado, Revelo, Trujillo y Gómez (2015); Chacón y Tapia (2017); Pineda (2007).
Artículos que tienen como tema central

la categoría "pareja sin hijos" y no

consideran este tipo de vínculo como una modalidad de familia.

Como se puede apreciar en la tabla anterior, la investigación sobre las parejas sin hijos es todavía incipiente, ya que solamente cinco investigaciones de las 35 recolectadas sobre familia, abordan de manera focalizada este modo de vinculación. Es cierto que hay una mayor cantidad de investigaciones que, por abordar las transformaciones de la familia en la actualidad, mencionan, en muchos casos sin desarrollar, la categoría de "parejas sin hijos". A continuación se mostrarán los principales planteamientos teóricos en torno a las parejas sin hijos y su relación con el concepto de "familia".

Algunas investigaciones señalan con estadísticas la manifestación de esta nueva forma de vinculación que muestra, como aspecto más relevante, la ausencia del carácter reproductivo o de procreación asociado tradicionalmente a la familia, en especial, bajo las concepciones clásicas o nucleares sobre este sistema. Así, por ejemplo, la investigación de Juárez (2007) sobre las transformaciones de la familia, denota que para ese año, en España, las familias sin hijos correspondían a un 10.8 $\%$; mientras que en la Unión Europea correspondían a un $17.3 \%$ (p. 821). En una investigación más reciente, Guerrero (2016), quien también se encarga de analizar los cambios sociales con respecto a la familia, afirma que "España está a la cabeza con un $48.6 \%$ de hogares formados por una pareja con hijos, $y$ solo un $18 \%$ está constituido por pareja sin hijos" (p. 173). No es para nada insignificante el aumento de más de siete puntos porcentuales en esta modalidad de familia en tan solo 8 años.

Chacón y Tapia (2017), en una investigación sobre las mujeres que deciden no tener hijos, afirman que "según el informe Anual de Population Reference Bureau se advierte una disminución en el número de hijos por mujer, la tasa global de fecundidad a nivel mundial ha pasado de 4.7 hijos por mujer en 1970 a un promedio de 2.5 hijos en la actualidad [2005]" (p. 195). Sumado a ello, muestra una estadística según la cual, para el año 2006, el $47 \%$ de las mujeres chilenas en edad fértil y que no tenían hijos habían manifestado no desear tenerlos en el futuro (p. 195). Más adelante, ofrece otras estadísticas que pueden ser complementarias a las planteadas por Juárez (2007):

"En Europa las cifras son reveladoras: la tasa global de fecundidad media ha llegado a 1.5 hijos por mujer (Baird et al., 2010). De 
acuerdo al microcenso realizado en Alemania el año 2012, alrededor del $22 \%$ de las mujeres de entre 40 y 44 no ha tenido hijos. En la República Federal, las mujeres sin hijos llegaron al $23 \%$ en el mismo año (Centro Alemán de Información, 2013). Según el United States Census Bureau, para el año 2014, las mujeres de entre 40 y 44 años de edad que no han tenido hijos llega al $15.3 \%$ en Estados Unidos (USAID, 2014)". (p. 197)

La reducción de la fecundidad es mucho más extendida en China que, a pesar de haber partido de un intento de control de la natalidad a partir de una decisión estatal, las dificultades económicas, en especial para el cubrimiento de las pensiones, han implicado que el gobierno impulse nuevamente a la procreación. No obstante, los estudios de Wong (2015), Xu y Xia (2014) y Zhongxin, Zhan y Xizhe (2017) muestran que los porcentajes de familias sin hijos está en aumento a la par que reduce la tasa de fecundidad; Zhongxin, Zhan y Xizhe (2017) afirman que "el número de niños por familia disminuyó de 1.48 en 1982 a 0.55 en 2015 " (p. 3). Esto es debido, según los autores, a "la aparición de un gran número de familias no tradicionales, como familias exclusivamente ancianas, familias de nidos vacíos, familia de abuelos, familia DINK, familia soltera mayor y familia monoparental" (p. 6).

La mayoría de los artículos citados en los últimos párrafos no profundizan en las razones que sustentan el crecimiento de las parejas sin hijos y la reducción de la fecundidad. Chacón y Tapia (2017) sí ofrecen una lectura en rigor planteando como principal punto de análisis la modificación del lugar de la mujer en la sociedad desde inicios del siglo XX. Según las autoras, la reducción de la fecundidad está dada porque las mujeres han tenido una mayor inserción en el mercado laboral y su nivel de escolaridad ha aumentado, lo que ha dado lugar a cuestionamientos sobre la distribución tradicional de labores en el hogar y ha derivado, a la vez, en una transformación sobre los roles de género (p. 198).

Sumado a lo ya planteado al respecto en el apartado anterior, puede decirse que las modificaciones en torno al género han puesto en cuestión la obligación de que las mujeres sean madres para realizar su feminidad, lo cual ha invitado también a los hombres a repensar los imaginarios tradicionalmente asociados a su género (Gallardo et al., 2006). En consecuencia, es cada vez más notorio que en los vínculos conyugales actuales aparezcan formas de vinculación más equitativas y democráticas en las que las mujeres y los hombres pueden tomar decisiones consensuadas, por ejemplo, en torno a la procreación; para Chacón y Tapia (2017) esto ha dado lugar a una mayor apertura para la emergencia de familias sin hijos. Poner en cuestión la procreación parece inadecuado y más si se piensa en la conservación de la especie, pero como se ha planteado en el apartado anterior, las investigaciones muestran que el criterio biológico es tan solo un componente en la definición de la familia y no es menos definitorio que aquellos correspondientes al tejido discursivo que se configura en una cultura particular y en un momento histórico específico. En esa línea, Cadenas (2015) puntualiza que el problema central de la familia moderna no puede ser la procreación, sino más bien la filiación que pone en cuestión el criterio de consanguinidad:

"La filiación y no la procreación es parte de la familia, no solo porque la ciencia moderna ha deconstruido este principio de derecho natural, sino también porque es en la filiación y no la procreación donde reside el problema social (comunicacional) de la familia. Las familias monoparentales o los matrimonios sin hijos muestran, adicionalmente, que la familia moderna requiere en la dimensión social solamente de roles complementarios mínimos para su autopoiesis." (pp. 37-38)

Este tipo emergente de familia ha dado lugar al surgimiento de algunas categorías que le son subsidiarias tales como DINK, Childfree, o NoMo (Chacón y Tapia, 2017; Pineda, 2007). La sigla DINK significa en inglés "double-income; no kids" (sueldo doble sin 
hijos), lo cual hace alusión explícita al factor económico que incide en la conformación de este tipo de familias; el término "Childfree" significa literalmente "sin hijos"; mientras que la sigla NoMo significa "No Mother", para hacer alusión tanto a las mujeres que no desean tener hijos como a las mujeres que, por razones biológicas, no pueden ser madres (Chacón y Tapia, 2017).

Finalmente, una de las investigaciones más llamativas fue la realizada por Cárdenas, Coronado, Revelo, Trujillo y Gómez (2015), ya que realiza un abordaje muy minucioso desde una perspectiva psicológica sobre esta modalidad de familia explorando la dinámica relacional de siete parejas que decidieron no tener hijos. Los autores consideran que las familias sin descendencia están surgiendo como una nueva forma de organización dentro de las tipologías familiares; esta organización cuenta con una estructura y dinámica que le es propia, razón por la cual la definen como un:

"Sistema conyugal de dos personas sin distinción de género en convivencia permanente y voluntaria, que comparten un mismo lecho sin importar el rito que manifieste su unión. Cumple funciones de protección, compañía y apoyo mutuo. Incluye proyectos tanto individuales como compartidos y un sistema de valores y creencias que soporta su identidad. Se preserva en ella, la individuación e implica el reconocimiento del otro". (Cárdenas et al., 2015, p. 145)

Los mismos autores consideran además que la modernidad y la posmodernidad han traído consigo una redefinición de las relaciones de pareja y las relaciones familiares, por lo cual es común la emergencia de nuevas organizaciones que no dan continuidad a los patrones relacionales culturalmente hegemónicos. Esto también puede ser favorecido con la idea de que en la pareja posmoderna aparece "la renuncia a los hijos como mecanismo para mantener la comodidad y favorecer proyectos individuales de los miembros" (p. 147). En ese sentido, es evidente que los autores no consideran la procreación como un criterio para la configuración de una familia; para ellos, lo que define a una familia se encuentra dado por la presencia de funciones tales como "la nutrición relacional, la socialización, la protección y el apoyo a los proyectos y decisiones" (Cárdenas et al., 2015, p. 158).

Ahora bien, sobre la relación que se puede establecer en esta investigación documental entre las parejas sin hijos y el concepto de "familia", puede decirse que la mayoría de los artículos que se ocupan de las transformaciones sobre este sistema conciben a dicha forma de vínculo como una forma emergente de familia. De las 18 investigaciones citadas solamente tres no sitúan a las parejas sin hijos como una modalidad de familia pues, como en el caso de Garrido, Reyes y Torres (2008) o de Pérez (1995), que son investigaciones sobre las expectativas de las parejas y sobre las parejas sin hijos respectivamente, los autores nunca se refieren a este tipo de vinculación como un tipo de familia; esto puede deberse a que no hay un ejercicio de problematización del significado de "familia" en ninguno de los dos estudios. Por su parte, en la investigación de Bernal (2013) sobre las parejas LAT (parejas que tienen un vínculo afectivo, pero no conviven juntos), la separación de expresiones como "nuevas familias y nuevas parejas" (p. 176), da cuenta de una separación y no de una integración entre ambas categorías.

En cuanto a los demás artículos, algunos autores ofrecen tipologías de familia en las que aparecen de manera explícita las expresiones "parejas que eligieron no tener hijos" (Baeza, 2005, p. 37), "nuclear sin hijos" (González, 2011, p. 67), "familia conyugal sin hijos" (Palacio, 2009, p. 55) y "familias sin hijos" (Vega, 2010). En el caso de Benítez (2017), aunque no menciona explícitamente la categoría de parejas sin hijos, sí ofrece una definición de "familia" que la incluye al afirmar, citando a Alberdi, que "la familia está formada por dos o más personas unidas por el afecto, el matrimonio o la filiación, que viven juntos, ponen sus recursos económicos en común y 
consumen conjuntamente una serie de bienes en su vida cotidiana" (p. 66). Más adelante menciona: "los hijos -si hay- son también parte de la familia, pero estos pudieran llegar a faltar, algo que era impensado antes del cambio social producido por la llamada Revolución Sexual" (p. 66). En ese sentido, puede abstraerse según dichas afirmaciones que el autor no considera que la procreación sea un criterio indispensable para la conformación de una familia.

Desde el punto de vista del derecho, en el recorrido histórico que realiza Esborraz (2015) sobre las modificaciones constitucionales en torno a la familia, el autor hace alusión a la familia convivencial para referirse a la figura presente en muchas cartas magnas como unión libre o de hecho. Este tipo de unión no implica la presencia de hijos o el proyecto futuro de procrear, lo que da cuenta de que, en materia legislativa, las parejas sin hijos pueden considerarse como una tipología de familia.

\section{Conclusión}

Las investigaciones recolectadas muestran una evidente transformación en el concepto de "familia", en especial sobre la visión tradicional de una familia nuclear conformada por papá, mamá e hijos. Los artículos que apelan a datos estadísticos muestran una creciente tendencia a la conformación de hogares y familias que deciden no tener hijos, hecho vinculado según los investigadores a un profundo cuestionamiento favorecido por la revolución feminista a partir del siglo XX. Las mujeres han puesto en cuestión su lugar en la familia y en la sociedad, y al modo de una estructura en la cual los elementos son interdependientes, esta modificación ha favorecido el movimiento de otros elementos intervinientes como el género, las políticas, las legislaciones y por supuesto, la familia.

Como se pudo apreciar, la investigación sobre parejas sin hijos es todavía incipiente, por lo cual es importante una apertura investigativa hacia esta nueva forma de vincula- ción familiar que, como se ha expresado, ha tomado fuerza en la sociedad. Se hace necesario investigar, con mayor profundidad y desde perspectivas interdisciplinares, cuáles son las nuevas narrativas que las parejas están tejiendo alrededor de su elección de no tener hijos como su propia concepción sobre la familia. También se hace necesario estudiar demográficamente y desde el punto de vista de la economía las posibles consecuencias que pueda tener a futuro la reducción de la tasa de fecundidad a partir de ese nuevo tipo de configuración familiar para una región o país en particular.

Esta investigación se concentró en una lectura a modo de estado del arte sobre los artículos de investigación publicados relativos al objeto de indagación. Se sugiere, por lo tanto, realizar investigaciones que amplíen el material de análisis a fuentes como libros, tesis de maestría y tesis doctorales que puedan ofrecer otros análisis sobre este asunto.

El estudio comparativo de los artículos también permite concluir que la mayoría de investigaciones consideran a las parejas sin hijos como una tipología de familia. Según varios autores citados, esto ha incidido en los criterios que definían a la familia, pues por ejemplo el criterio biológico de consanguinidad, la presencia de un hombre y una mujer, o la necesidad de procreación ya no son indispensables en varias de las tipologías emergentes. Los autores reconocen la importancia de la conyugalidad y la filiación, el establecimiento de vínculos afectivos y de un apoyo mutuo que respete los proyectos individuales y favorezca el tejido de proyectos comunes sin renunciar a todas las aspiraciones particulares de sus integrantes. Los autores también resaltan el surgimiento de un carácter más equitativo y democrático que da lugar a la toma de decisiones consensuadas, en el que las tareas del hogar empiezan a distribuirse en función de la transformación de los roles de género.

Finalmente, algunos autores reconocen que en la actualidad no es posible hablar de una 
"familia ideal" en tanto las nuevas configuraciones obedecen a los valores y las creencias de sus integrantes; estos tejen en conjunto una narrativa sobre la familia que establece una dinámica particular que la diferencia de cualquier otra, lo cual hace que las familias en último término sean únicas. Para varios autores esto da lugar a una visión más pluralista de la familia a la cual debe responder el Estado, la ciencia y la cultura en general, con el fin de dar lugar a las nuevas necesidades que esta institución va demandando.

\section{Referencias bibliográficas}

Acevedo, L. (2011). El concepto de familia hoy. Franciscanum. Revista de las Ciencias del Espíritu, LIII(156), 149-170. Recuperado de http://www.scielo.org.co/ $\mathrm{pdf} / \mathrm{frcn} / \mathrm{v} 53 \mathrm{n} 156 / \mathrm{v} 53 \mathrm{n} 156 \mathrm{a} 06 . \mathrm{pdf}$

Alberdi, I. (2016). El retraso de la procreación y el mantenimiento de la juventud. Metamorfosis. Revista del Centro Reina Sofía sobre Adolescencia y Juventud, 0. Recuperado de http://revistametamorfosis.es/ index.php/metamorfosis/article/view/8

Álvarez, L. (2008). La familia, desarrollo y cambio social. Claves para un estudio interdisciplinario. En-Claves, 2(4). Recuperado de http://www.scielo.org. $\underline{\mathrm{mx} / \text { scielo.php?script }=\text { sci arttextypi- }}$ $\mathrm{d}=\mathrm{S} 1870-879 \mathrm{X} 2008000200001$

Baeza, S. (2005). Familia y género: Las transformaciones en la familia y la trama invisible del género. Praxis Educativa, 9. Recuperado de http://www.redalyc.org/ comocitar.oa?id=153120512004

Benítez, M. (2017). La familia: Desde 10 tradicional a lo discutible. Centro de Estudios Demográficos de la Universidad de la Habana, 13(26). Recuperado de http:// scielo.sld.cu/scielo.php?script $=$ sci arttextypid=S1817-40782017000200005

Bernal, I. (2013). "Juntos aunque separados". Parejas LAT en la ciudad de Medellín. Revista Facultad Trabajo Social, 29. Recuperado de https://revistas.upb.edu.co/ index.php/trabajosocial/article/view/2333

Builes, M. y Bedoya, M. (2008). La familia contemporánea: relatos de resiliencia y salud mental. Revista Colombiana de Psiquiatría, 37(3). Recuperado de http://www.scielo.org.co/pdf/rcp/v37n3/ v37n3a05.pdf

Cadenas, H. (2015). La familia como sistema social: Conyugalidad y parentalidad. Revista Mad. Revista del Magíster en Análisis Sistémico Aplicado a la Sociedad, 33. Recuperado de http://www.redalyc.org/ articulo.oa? $\mathrm{id}=311241654004$

Cadenas, H. y Urquiza, A. (2016). Naturaleza y contingencia en la familia moderna. Espacio Abierto. Cuaderno Venezolano de Sociología, 25(3). Recuperado de http://www.redalyc.org/articulo. oa?id=12249678005

Cárdenas, M., Coronado, D., Revelo, M., Trujillo, S. y Gómez, J. (2015). Dinámica relacional de familias que deciden no tener descendencia. Revista de Psicología Universidad de Antioquia, 7(1). Recuperado de http://aprendeenlinea.udea.edu. co/revistas/index.php/psicologia/article/ view/25268/20878

Chacón, F. y Tapia, M. (2017). "No quiero tener hijos (as)... continuidad y cambio en las relaciones de pareja de mujeres profesionales jóvenes". Polis [En línea], 46. Recuperado de http://journals.openedition. org/polis/12339

Corbi, M. (1996). Religión sin religión. Madrid: Servicios Koinonía.

Crespo, F. (2015). Hacia la familia conyugal, aproximación a los discursos periodísticos en los siglos XVIII y XIX. Dos Puntas, 12. Recuperado de https://dialnet.unirioja.es/ descarga/articulo/5494844.pdf

Di Brienza, M. (2007). Modalidad en las uniones conyugales en Venezuela, continuidad y cambios. Revista Temas de coyuntura, 55. Recuperado de http://revistasenlinea.saber.ucab.edu.ve/temas/index.php/ temasdecoyuntura/article/view/1247/1113

Esborraz, D. F. (2015). El concepto cons- 
titucional de familia en América Latina. Tendencias y proyecciones. Revista De Derecho Privado, 29. http://doi. org/10.18601/01234366.n29.02

Fernández, C. (2017). “Amor, matrimonio y procreación: sobre la teoría del amor de José Ingenieros". En O. Rey Castelao y P. Cowen (Eds.), Familias en el Viejo y el Nuevo Mundo (pp. 341-366). La Plata: Universidad Nacional de La Plata. Colección Hismundi.

Gallardo, G., Gómez, E., Muñoz, M. y Suárez, N. (2006). Paternidad: Representaciones Sociales en Jóvenes Varones Heterosexuales Universitarios sin Hijos. Psykhe, 15(2). Recuperado de http://www.psykhe. cl/index.php/psykhe/article/view/315/295

Gallego,A. (2012). Recuperación crítica de los conceptos de familia, dinámica familiar y sus características. Revista virtual Universidad Católica del Norte, 35. Recuperado de http://revistavirtual.ucn.edu.co/index. php/RevistaUCN/article/view/364/679

Garrido, A., Reyes, A., Torres, L. y Ortega, P. (2008). Importancia de las expectativas de pareja en la dinámica familiar. Enseñanza e Investigación en Psicología, 13(2). Recuperado de http://www.redalyc.org/articulo. oa?id=29213203

Gómez, M., Galeano, C. y Jaramillo, D. (2015). El estado del arte: una metodología de investigación. Revista Colombiana de Ciencias Sociales, 6(2). Recuperado de http://www. funlam.edu.co/revistas/index.php/RCCS/ article/view/1469/pdf 26

González, M. (2009a). Nuevas familias, nuevos retos para la investigación y la educación. Cultura y Educación, 21(4). https://doi. org/10.1174/113564009790002409

González, M. (2009b). Familia, un actor con historia propia. Cuadernos de Teología, 1. Recuperado de http://146.83.115.167/index. php/teologia/article/view/1031/827

González, J. (2011). Familia hoy: elementos para la discusión. Studiositas, 1(6). Recuperado de https://dialnet.unirioja.es/descarga/articulo/4459916.pdf
Grau, C. y Fernández, M. (2015). Relaciones de parentesco en las nuevas familias. Disociación entre maternidad/paternidad biológica, genética y social. Gazeta de Antropología, 31(1). Recuperado de http://www.gazeta-antropologia.es $/ ? \mathrm{p}=4660$

Guba, E. y Lincoln, Y. (2002). Paradigmas en competencia en la investigación cualitativa. En C. Denman y J. Haro (Comps.), Antología de métodos cualitativos en investigación social (pp. 113-145). Hermosillo, Sonora: El Colegio de Sonora.

Guerrero, J. (2016). La familia: realidades y cambio social. La Razón Histórica, 33. Recuperado de https://dialnet.unirioja.es/servlet/ articulo? codigo $=6328762$

Guevara, R. (2016). El estado del arte en la investigación: ¿análisis de los conocimientos acumulados o indagación por nuevos sentidos? Folios, 44. Recuperado de http://www.scielo. org.co/scielo.php?script=sci arttextypi$\mathrm{d}=$ S0123-48702016000200011ylng=enytlng

Gutiérrez, R., Díaz, K. y Román, R. (2016). El concepto de familia en México: una revisión desde la mirada antropológica y demográfica. Ciencia. Ergo-sum, Revista Cientifica Multidisciplinaria de Prospectiva, 23(3). Recuperado de https://dialnet.unirioja.es/servlet/articulo?codigo $=5713921$

Hoyos, C. (2000). Un modelo para investigación documental. Guía teórico-práctica sobre construcción de estados del arte. Medellín: Señal.

Juárez, M. (2007). La familia: algunos cambios sociales significativos. Miscelánea Comillas, 65(127). Recuperado de https://revistas.comillas.edu/index.php/miscelaneacomillas/article/ viewFile/7354/7200

Lopera, J. (2016). Paternidad o procreación responsable: Iglesia católica, Acción Cultural Popular y control de la natalidad en Colombia (1964-1978). Historia y Sociedad, 31. Recuperado de http://www.scielo.org.co/pdf/hiso/ n31/0121-8417-hiso-31-00235.pdf

Lopera, J., Ramírez, C., Zuluaga, M. y Ortiz, J. (2010). El método analítico. Medellín: Centro de Investigaciones Sociales y Humanas de la Universidad de Antioquia. 
Machado, J. A. (2014). La transformación del concepto constitucional de familia. Alcances a una problemática teórica. Díkaion. Revista de Fundamentación Jurídica, 23(1), 93-133. Recuperado de http://www.scielo.org.co/scielo. php?pid=S0120-89422014000100005yscrip$\mathrm{t}=\mathrm{sci}$ abstractytlng $=\mathrm{es}$

Martín, M. y Echavarría, N. (2017). Narrativas sobre la organización familiar durante el desempleo masculino. Interdisciplinaria, 34(2). Recuperado de http://www.scielo.org. ar/pdf/interd/v34n2/v34n2a03.pdf https://doi. org/10.16888/interd.2017.34.2.3

Martínez, C. (2015). De nuevo la vida. El poder de la no violencia y las transformaciones culturales. Bogotá: Trillas.

Martínez, L. y Fernández, S. (2017). Nuevas familias e intervención social: aproximación conceptual a las nuevas modalidades familiares monoparentales. Documentos de Politica Social. Historia, Investigación y desarrollo, 40(5). Recuperado de https://www.researchgate.net/ profile/Antonio_Martinez-Martinez/publication/324497389 Nuevas familias e intervencion_social_aproximacion_conceptual_a las_nuevas modalidades familiares monoparentales/links/5ad087c3a6fdcc8784121dea/ Nuevas-familias-e-intervencion-social-aproximacion-conceptual-a-las-nuevas-modalidades-familiares-monoparentales.pdf

Moreno, A. (2010). La emergencia de una nueva concepción de la familia entre las y los jóvenes españoles. Revista de Estudios de Juventud, 90. Recuperado de https://dialnet.unirioja.es/ servlet $/$ articulo? codigo $=3651088$

Nieri, L. (2017). Transición y construcción de la paternidad. Revista interdisciplinaria. Revista de Psicología y ciencias afines, 34(2). Recuperado de https://www.researchgate.net/publication/330264823_Transicion_y_construccion de la paternidad. https://doi.org/10.16888/ interd.2017.34.2.11

Oliva, E. y Villa, V. (2014). Hacia un concepto interdisciplinario de la familia. Revista de Justicia Juris, 10(1). Recuperado de http:// www.scielo.org.co/pdf/jusju/v10n1/v10n1a02. pdf
Palacio, C. (2009). Los cambios y transformaciones en la familia. Una paradoja entre lo sólido y lo líquido. Revista Latinoamericana de Estudios de Familia, 1. Recuperado de http://vip.ucaldas.edu.co/revlatinofamilia/ downloads/Rlef1_3.pdf

Pérez, F. (1995). Las parejas sin hijos en Portugal y España. Revista Española de Investigaciones Sociológicas, 70. http://www.reis.cis.es/REIS/ PDF/REIS 070 06.pdf

Pineda, C. (2007). ¿Las parejas sin hijos viven mejor? Contenido. Issue, 525, 29-32.

Uribe, J. (2011). La investigación documental y el estado del arte como estrategias de investigación en ciencias sociales: estrategias de investigación. En P. Páramo (Comp.), La investigación en ciencias sociales (pp. 195-210). Bogotá: Universidad Piloto.

Vásquez, C. (2005). Las nuevas tipologías familiares y los malestares interrelacionales que se suscitan en ellas. Revista Virtual Universidad Católica del Norte, 14. Recuperado de http:// revistavirtual.ucn.edu.co/index.php/RevistaUCN/article/view/251/476

Vega, Y. (2010). El nuevo rostro de la familia: cuadros de una exposición. Anuario de la Facultad de Derecho, 28. Recuperado de https://dialnet.unirioja.es/servlet/articulo?co$\underline{\text { digo }=3438248}$

Vélez, O. y Galeano, M. (2002). Investigación cualitativa. Estado del arte. Facultad de Ciencias Sociales y Humanas. Centro de Investigaciones Sociales y Humanas CISH. Medellín: Editorial Universidad de Antioquia.

Walters, K., Cintrón, F. y Serrano, I. (2006). Familia Reconstituida. El Significado de "Familia" en la Familia Reconstituida. Psicología Iberoamericana, 14(2). Recuperado de http://www. redalyc.org/pdf/1339/133920321003.pdf

Wong, D. (2015). Asexuality in China's sexual revolution: Asexual marriage as coping strategy. Sexualities, 18(1-2), 100-116. https://doi. org/10.1177/1363460714544812

Xu, A. y Xia, Y. (2014). The changes in Mainland Chinese Families During the Social Transition: A Critical Analysis. Journal of Comparative Family Studies, XLV (1). http://digitalcom- 
mons.unl.edu/famconfacpub/91/

Zhongxin, S., Zhan H. y Xizhe, P. (2017). The impact of changes in china's family patterns on family pension functions. International Journal of Health Planning and Management, 32(3), 1-12. https://doi.org/10.1002/hpm.2436

Recibido: 3 de diciembre de 2018 Aceptado: 19 de agosto de 2020 